\title{
Extrapolation of Black- and Volatile-oil Fluid Properties with Application to Immiscible/Miscible Gas Injection
}

\author{
Bahareh Nojabaei, University of Wyoming; Russell T. Johns, The Pennsylvania State University
}

\begin{abstract}
Black-oil fluid properties are determined by lab measurements or can be calculated through flash calculations of the reservoir fluid. Allowing for variable bubble-point pressures in black- or volatile-oil models requires a table of fluid properties be extended above the original bubble-point. Reservoir simulation accuracy, however, may be affected by discontinuities in the input data and poor predictions of extrapolated fluid properties. Common practice is to add surface gas to the original oil in the lab and increase the pressure to reach a new bubble-point. Another approach is to use linear extrapolation of oil and gas $K$-values with pressure on a log-log plot, where $K$-values are equal to 1.0 at a pseudo-critical or convergence pressure. The latter approach results in discontinuities in the phase behavior.

We calculate continuous black-oil fluid properties above the original bubble-point by adding a fraction of the equilibrium gas at one bubble-point pressure to achieve a larger bubble-point pressure. This procedure continues until a critical point is reached at the top of the pseudocomponent pressure-composition diagram. Unlike other methods commonly used or recently proposed, the approach provides a smooth and continuous pressure-composition curve to the critical point. The model further allows for reinjection of produced gas, methane, or $\mathrm{CO}_{2}$ to increase oil recovery for both volatile and black oils. Further, the approach allows the use of black-oil or volatile-oil properties for tight rocks where capillary pressure alters the saturation pressures by decreasing the bubble-point pressure or increasing the dew-point pressure. Bubble-point pressure in the new model is a function of both capillary pressure (effective pore radius) and gas content. The phase behavior is illustrated using ternary diagrams for up to four components (water, oil, gas, and $\mathrm{CO}_{2}$ or $\mathrm{CH}_{4}$ ) and three phases (aqueous, oleic, gaseous) to allow for miscible and immiscible injection of various gases. The new phase behavior could be easily incorporated in a compositionally-extended black- or volatile-oil simulator. The approach could also be used to model gas condensate reservoirs with or without gas injection and capillary pressure. Finally, 1-D slim-tube simulations are made with the extrapolated black oil fluid properties to estimate minimum miscibility pressure (MMP) to demonstrate the applicability of the approach. Shocks occur in the 1-D displacement consistent with a compositional simulator.
\end{abstract}

\section{Introduction}

Black oil simulation is widely used in reservoir simulation because it is fast and more robust than compositional simulation and is reasonably accurate when little mass transfer between phases occurs. Tabulation of black oil fluid properties from either measured data or numerical flash calculations are made prior to performing simulation. These fluid properties should be determined to reflect accurately the change of fluid properties with pressure during pressure depletion, gravity segregation, or gas injection.

Black oil fluid properties are typically estimated from constant composition expansion (CCE), differential liberation (DL) and separator tests. For CCE tests, the oil composition is constant, while for DL tests the liquid composition changes with pressure as liberated gas is removed from the cell during pressure decline steps (Dandekar, 2013).

Besides using experiments, flash calculations using cubic equations-of-state (EOS) can be used to calculate black-oil properties such as bubble-point pressures, formation volume factors, densities, viscosities, solution gas-oil ratio and volatile oil-gas ratio in the case of volatile oils or gas condensates. Many of these parameters are typically determined as functions of pressure and the gas content (measured by the solution gas-oil ratio for undersaturated fluids). The flash calculation approach, however, relies on accurate cubic equation-of-state modeling.

Compositionally extended black oil models offer significant advantages in terms of solution robustness, especially when bubble-point pressure may change owing to changes in gas content (Wong et al. 1987) and when capillary pressure impacts the phase behavior (Nojabaei et al 2014). Nojabaei et al. (2013) showed that the bubble-point of oil is lowered by large capillary pressure in tight unconventional rocks, while the dew-point pressure is typically increased. The compositional formulation of a black oil, volatile oil, or gas condensate is more robust during gas phase appearance or disappearance since the components of the Jacobian in a fully implicit scheme are unchanged for two and three-phase flow. For black oils, the principle unknowns in the variable bubble-point formulation are oil pressure, overall gas composition and water saturation. These unknowns are unchanged using the compositional approach when a gas phase (or liquid phase) appears or disappears. Flash calculations in the compositional approach are non-iterative and are based on $K$-values generated from the input blackoil data. The input fluid properties to the compositionally-extended black oil simulation are oil and gas densities and viscosities, solution gas-oil ratio, and volatile oil-gas ratio.

To allow for variable bubble-point pressure in reservoir simulation, black- and volatile-oil fluid properties should be available above the original bubble-point pressure. There are different ways to extrapolate these properties above the original bubble point pressure. One approach is to use a visually corrected extrapolation that honors the expected shape of the fluid properties curve. MacCain and Spivay (1999) proposed a similar approach; they visually extrapolated the black oil fluid properties and then corrected their extrapolated curves so that the theoretical and experimental relationships between these properties are honored. They did not extend, however, the extrapolated fluid properties to a critical point.

Singh et al. (2007) extrapolated the data to higher saturation pressures by including a convergence or critical pressure at 
which phase properties became identical. The linear log-log relationship between surface gas and oil $K$-values with pressure allowed them to extrapolate the fluid properties so that $K$-values are equal to 1.0 at the convergence pressure.

The extrapolated black-oil properties can also be measured in the lab by combing produced gas with the dead oil. After addition of more gas than initially present in the original live oil, the pressure is increased until the gas disappears at the new bubble point. Montel and Quettier (2004) stated that for the case of gas injection in an undersaturated oil reservoir, the extrapolation of black oil properties above the initial saturation pressure should be done by adding increasing quantities of injection gas with initial oil.

The problem with these current methods is that they can produce discontinuities in the black oil properties with clearly observable inaccuracies in the PX diagrams of the two pseudocomponent models. Whitson and Sunjerga (2012) stated that the best way to extrapolate saturation properties for oil rich shales is to add increments of the incipient phase from each new elevated saturation pressure. That is, they proposed to add equilibrium gas with bubble-point oil at each higher pressure until the critical pressure was reached at constant reservoir temperature. In their paper, however, they only added gas in one discontinuous step instead of a more differential approach. At the critical point, they showed that the solution gas-oil ratio is equal to the inverse of the volatile oil-gas ratio when the same units are used. Oil viscosity and gas viscosity were therefore equal at the critical point in their approach.

In this paper, we extend the approach by Whitson and Sunjerga (2012) by adding bubble-point gases in smaller increments of pressure using the Peng-Robinson equation-of-state (Peng and Robinson, 1976). More importantly, we show how to include simultaneously the effect of capillary pressure and gas content in the oil on the extrapolated fluid properties and $K$-values. The results give continuous black- and volatile-oil properties for tight and conventional reservoirs above the original saturation pressures. Interfacial tension is modeled with the Macleod-Sugden correlation, which gives zero interfacial tension at the critical point. Pressure-composition plots of Bakken oil are presented for three different effective pore sizes and are used to represent compositionally-extended black-oil phase behavior on a ternary diagram where water is included as a separate component. A fourth component (methane or $\mathrm{CO}_{2}$ ) is added to the black- or volatile-oil phase behavior to demonstrate how the new approach could be used to model miscible and immiscible gas injection more accurately, compared to previous models that assume first-contact miscibility (e.g. Todd and Longstaf, 1972; Shehbaz and Hoffman, 2009; and Dong and Hoffman, 2012). We then compare the MMP from the pseudo-binary or ternary phase behavior to the calculated minimum miscibility pressures (MMPs) based on a cubic equation-of-state fluid characterization. Last, slim-tube simulation for Bakken oil is performed using the compositionally-extended black oil model to obtain the MMP for displacement of this oil by produced gas.

\section{Methodology}

In this section, we first review the methodology used by Nojabaei et al. (2013) to calculate the effect of capillary pressure on the phase behavior and fluid properties. We then use that approach to show how the black- or volatile-oil data can be extended for variable bubble-point formulation using a compositionally-extended approach where the oil composition is altered as gas mixes with it. The methodology is demonstrated using the Bakken reservoir fluid at a reservoir temperature of $240^{\circ} \mathrm{F}$ (Nojabaei et al. 2013).

\subsection{Calculation and Extrapolation of Fluid Properties}

Nojabaei et al. (2013) performed flash calculations that included the effect of capillary pressure on the phase behavior. We use the same approach here, but extend the calculations to the case where bubble-point pressure can also vary according to changes in the oil composition. Fluid properties including the PT two-phase envelope are calculated using the PengRobinson equation of state (Peng and Robinson, 1976) for effective pore sizes varying from $10 \mathrm{~nm}, 30 \mathrm{~nm}$, to infinitely large, where an infinite pore size corresponds to zero capillary pressure. Oil pressure is assumed to be the reference phase pressure, while gas pressure is calculated using the Laplace equation given below,

$P_{g}=P_{o}+\frac{2 \sigma}{r}$

Phase equilibrium is achieved when the component fugacities of the gas and oil phases are equal. That is,

$f_{i}^{o}\left(T, P_{o}, x_{1}, x_{2}, \ldots, x_{N_{C}}\right)=f_{i}^{g}\left(T, P_{g}, y_{1}, y_{2}, \ldots, y_{N_{C}}\right)$.

We use the flash calculation approach to determine the black- and volatile-oil properties, where the EOS model has been tuned to standard PVT data. First, the pressure is reduced in a constant composition expansion test to calculate oil and gas densities and viscosities. For pressures higher than the original bubble-point pressure, the reservoir fluid is a single phase liquid and the oil composition does not change. Above the bubble-point pressure, oil densities and viscosities are nearly linear with pressure and the solution gas-oil ratio is constant. To calculate solution gas-oil and volatile oil-gas ratios at each pressure below the original bubble-point pressure, we flash the overall composition at that pressure to obtain equilibrium 
liquid and gas compositions. The liquid composition is then flashed at standard conditions to calculate the solution gas-oil ratio, while the equilibrium gas composition is flashed to obtain the volatile oil-gas ratio. Solution gas-oil ratio is the volume of gas at standard condition that evolves from the oil divided by the volume of oil at standard condition (SCF/STB). The volatile oil-gas ratio is the volume of oil at standard condition that condenses from the gas divided by the volume of gas at standard condition (STB/MMSCF). These fluid properties are functions of oil pressure and solution gas content in the standard black- or volatile-oil model, but here they are also a function of capillary pressure.

To extrapolate the black oil fluid properties to pressures above the original bubble-point pressure, the overall composition of Bakken oil is adjusted by diluting the oil with various amounts of equilibrium gas. In the first step, the gas composition used for the dilution is the gas in equilibrium with the oil at the original bubble-point pressure. This gas composition is most easily calculated using a negative flash using a cubic equation-of-state, such as Peng-Robinson EOS. The new overall composition of the fluid that results from mixing a certain fraction of original oil with its equilibrium gas is given by,

$z_{\text {new }}=(1-\alpha) z_{\text {old }}+\alpha y_{\text {old }}$

where $\alpha$ is the dilution factor and $y$ the equilibrium gas composition. Typical values used here are 0.1 for $\alpha$, although we vary this value to show its impact on the derived phase behavior.

In the second step, this new overall composition is flashed and pressure increased until gas disappears at a new elevated bubble-point pressure. At the new bubble-point pressure, the fluid is again "negatively" flashed to find the new equilibrium gas composition. Eq.(3) is then used to determine a new overall composition. This procedure is repeated to obtain larger bubble-point pressures. The procedure ensures that the critical point will be at the top of the two-phase zone on the pressurecomposition diagram because the liquid and vapor compositions will eventually approach each other as pressure is increased.

Figures 1 demonstrates the approach for seven new bubble-point pressures and their corresponding oil density and solution gas-oil ratios. The densities for each new oil composition approach each other at the critical point near a pressure of 4,325 psia. The effect of capillary pressure is not included in this PX diagram, although we will do so later in the results section. Figures 1 shows that the curves do not exactly coincide for pressures below the original bubble-point pressure. This is because the process shown here is discrete in that seven new overall compositions were determined using $\alpha=0.1$ in Eq. (3). The curves will coincide exactly below the bubble-point pressure for infinitesimal additions of gas, that is, with $\alpha$ near zero.
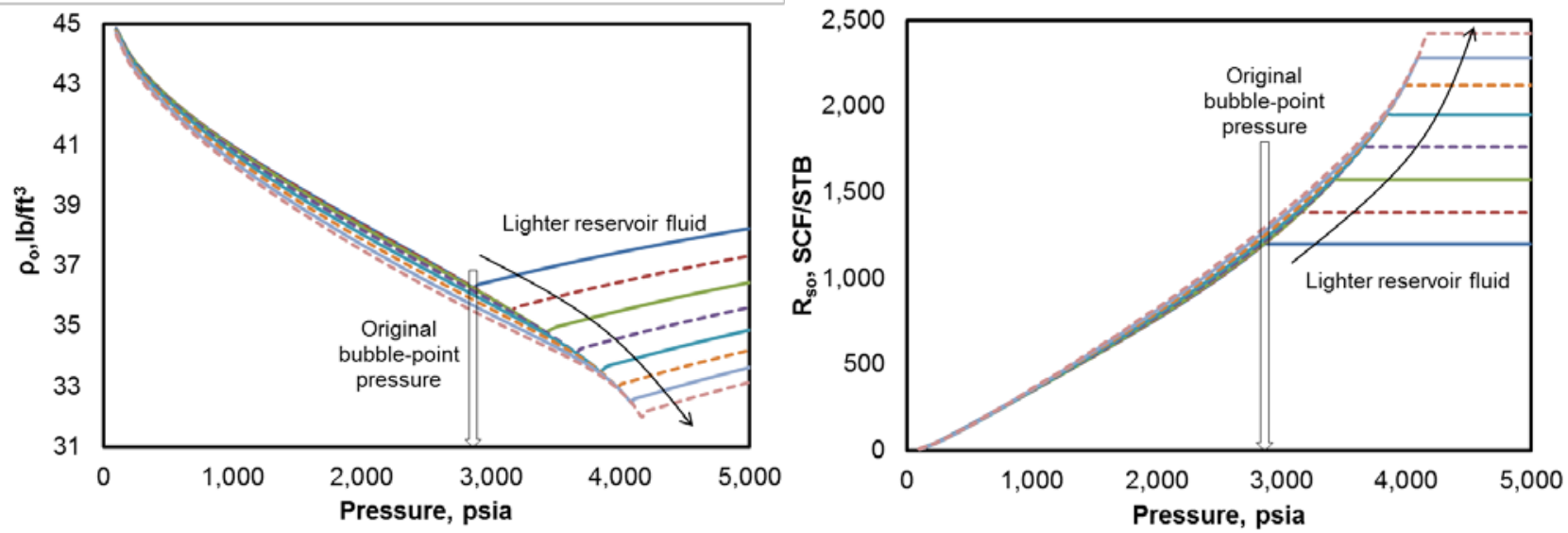

Figure 1- Extrapolation of oil density and solution gas-oil ratio by adding variable composition bubble-point gas from the last pressure.

The approach outlined above produces continuous black- or volatile-oil data for small $\alpha$. There are other possible ways to make the PX diagram, but these are not physically consistent. For example, the oil composition could be changed by adding a constant composition of gas at the original bubble point to the reservoir fluid. Figures 2, however, shows that this approach is incorrect because at higher pressures the curves intersect and do not converge to a single critical point. This is expected for mixing of real multicomponent fluids where the critical point along that dilution line does not occur at the top of the PX diagram.

When capillary pressure is present, the approach we outlined here must maintain consistency at the critical point. To extrapolate the fluid properties when capillary pressure affects the phase behavior also, the same equilibrium gas composition at the bubble point is added to the oil composition based on the compositions found assuming zero capillary pressure. This preserves the same overall compositions with and without capillary pressure and will give the same critical point no matter the effective pore size. At the critical point, the phase behavior is no longer a function of capillary pressure. 

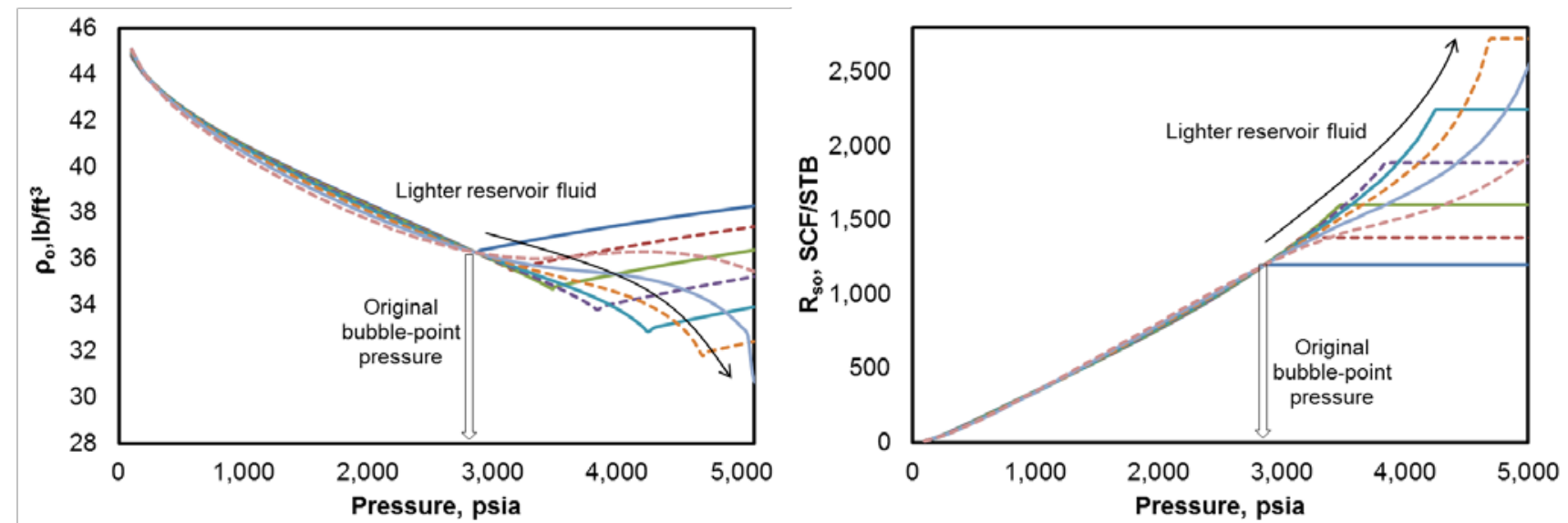

Figure 2- Extrapolation of oil density and solution gas-oil ratio by adding fixed composition bubble-point gas. This approach is incorrect.

\subsection{Calculation of $K$-Values and Oil and Gas Mass Fractions Using Black-Oil Fluid Properties}

The compositional-extended approach uses $K$-values calculated from the black- or volatile-oil fluid properties. The component $K$-values between the vapor and oleic phases at each pressure are explicitly calculated based on Eqs. (4) and (5).

$$
\begin{aligned}
& K_{o}=\frac{\omega_{o g}}{\omega_{o o}}=\frac{\left(R_{v} / 10^{6}\right)\left(5.615 \rho_{o s c}\right)\left(5.615 \rho_{o s c}+R_{s o} \rho_{g s c}\right)}{5.615 \rho_{o s c}\left(\left(R_{v} / 10^{6}\right)\left(5.615 \rho_{o s c}\right)+\rho_{g s c}\right)} \\
& K_{g}=\frac{\omega_{g g}}{\omega_{g o}}=\frac{\left(5.615 \rho_{o s c}+R_{s o} \rho_{g s c}\right)}{R_{s o}\left(\left(R_{v} / 10^{6}\right)\left(5.615 \rho_{o s c}\right)+\rho_{g s c}\right)}
\end{aligned}
$$

$K_{o}$ is defined as the mass fraction of oil component in the vapor phase divided by the mass fraction of oil component in the oleic phase, while $K_{g}$ is the mass fraction of the gas component in the vapor phase divided by the mass fraction of gas component in the oleic phase. These two equations are obtained by conserving component mass in the reservoir and at surface conditions and by taking oil and gas phases at the surface to consist of purely oil and gas pseudocomponents, respectively. Mass fractions of a given phase must also sum to one. Thus, the phase mass fractions, and hence $K$-values, are determined without iteration.

The gas and liquid phase compositions are obtained by rearrangement of Eqs. (4) and (5) along with the relationship that mass fractions must sum to 1.0 in a given phase. That is, the mass of gas component in each phase becomes,

$$
\begin{aligned}
& \omega_{g g}=\frac{\rho_{g s c}}{\left(R_{v} / 10^{6}\right)\left(5.615 \rho_{o s c}\right)+\rho_{g s c}} \\
& \omega_{g o}=\frac{R_{s o} \rho_{g s c}}{5.615 \rho_{o s c}+R_{s o} \rho_{g s c}} .
\end{aligned}
$$

At the critical point, $K$-values should be one, meaning that component liquid and gas mass fractions are equal. This fact coupled with the requirement that interfacial tension is zero at the critical point are used to examine the consistency of the extrapolated fluid properties near the critical point.

\section{Results and Discussion}

The Bakken reservoir fluid characterization at $240^{\circ} \mathrm{F}$ is used for all examples in this section as described in Nojabaei et al. (2013). Although only one reservoir fluid type is analyzed here, our approach can be applied to any other reservoir fluids without restriction. The change in bubble-point pressure can be even greater for other reservoir fluids as Siripatrachai et al. (2016) concluded for the Eagle Ford fluid compared to Bakken.

Here, we first consider bubble-point pressure as a function of both gas content in the oil and capillary pressure. Figure 3 shows the solution gas-oil ratio and its extrapolation for simultaneous changes in gas content (solution gas-oil ratio above 
bubble-point pressure) and the effective pore size (capillary pressure). As shown, the bubble-point pressure decreases when capillary pressure increases while the bubble-point pressure increases as the gas content in the oil increases. The original bubble-point pressure for the case of no capillary pressure is 2,860 psia, while the original bubble-point pressures for $30 \mathrm{~nm}$ and $10 \mathrm{~nm}$ effective pore sizes are 2,784 and 2,572 psia respectively. The difference in the solution gas-oil ratio owing to capillary pressure increases as the pressure is reduced.

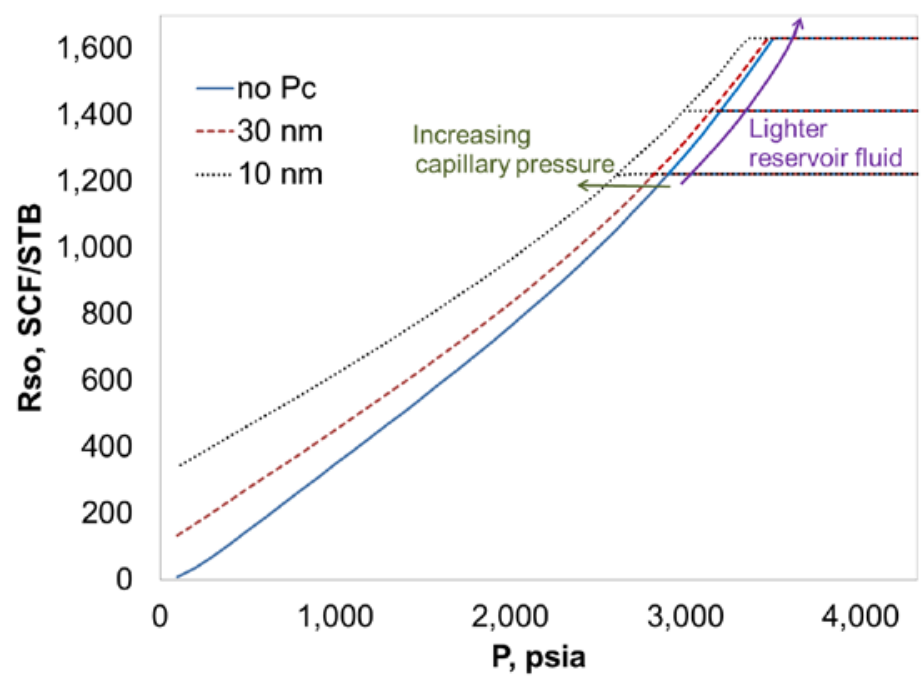

Figure 3- Solution gas-oil ratio at three different effective pore sizes and for three different gas contents to show the simultaneous change in extrapolated bubble-point pressure.

Figures 4 to 7 give various extrapolated black-oil properties and show that these properties converge as they should at the critical point where capillary pressure is zero.

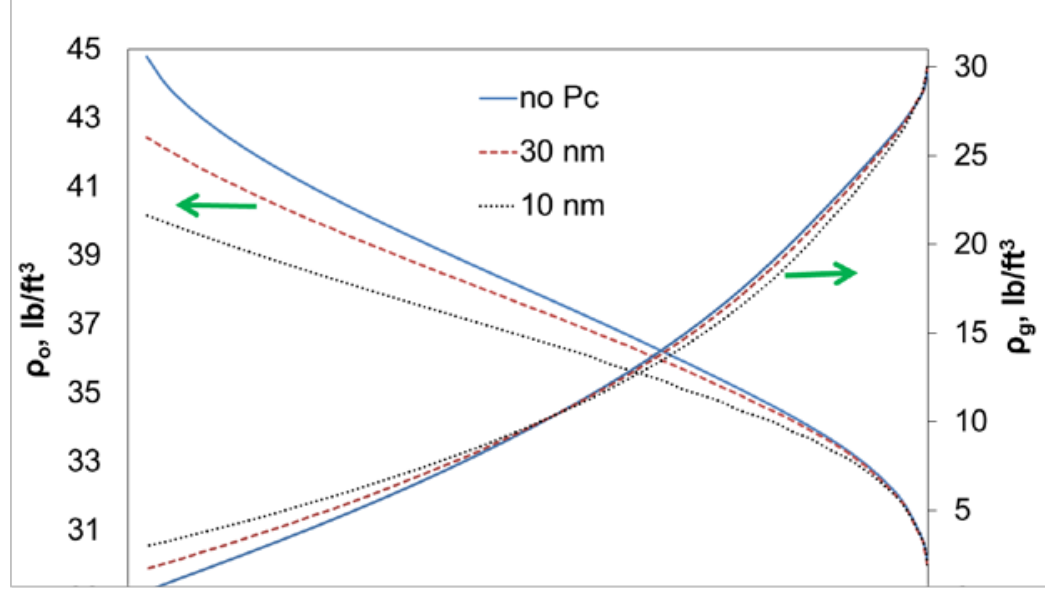

Figure 4- Oil and gas density change with oil pressure and effective pore radius up to the critical point at 4325 psia. 


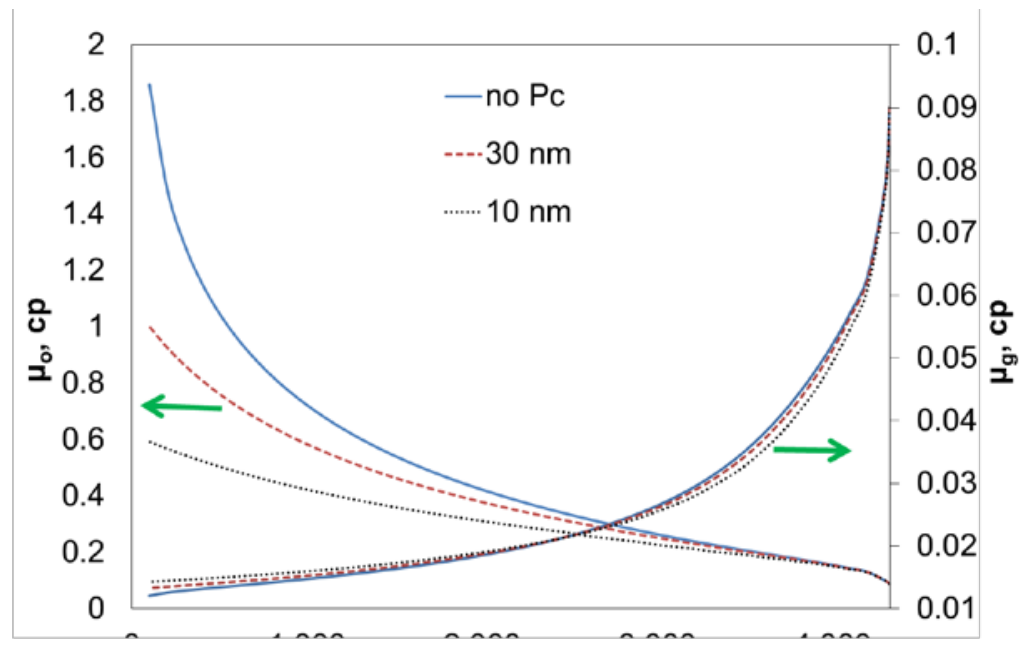

Figure 5- Oil and gas viscosity change with oil pressure and effective pore radius up to the critical point at 4325 psia.

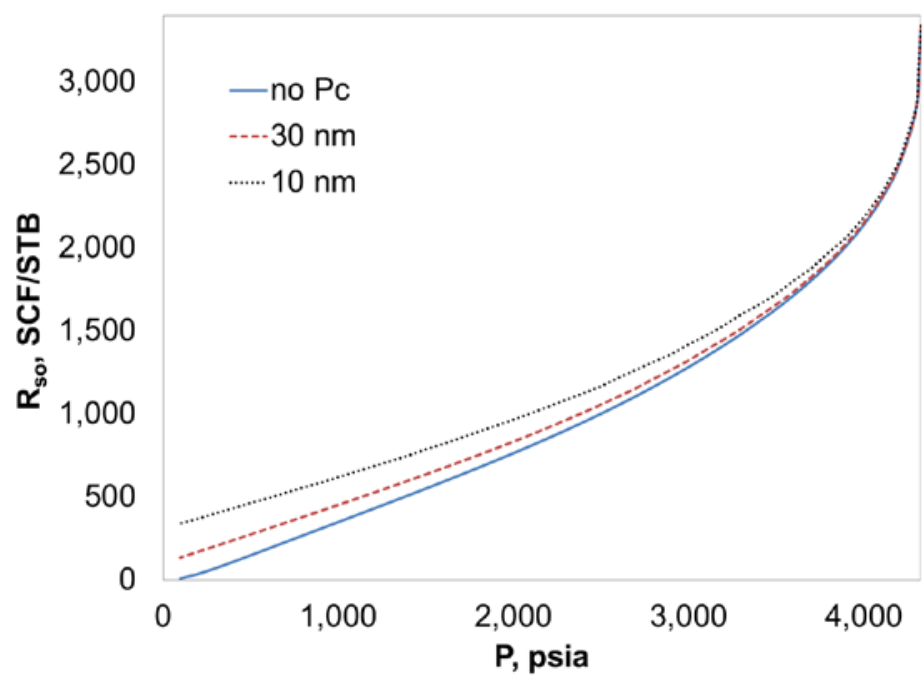

Figure 6- Solution gas-oil ratio change with oil pressure and effective pore radius up to the critical point at 4325 psia.

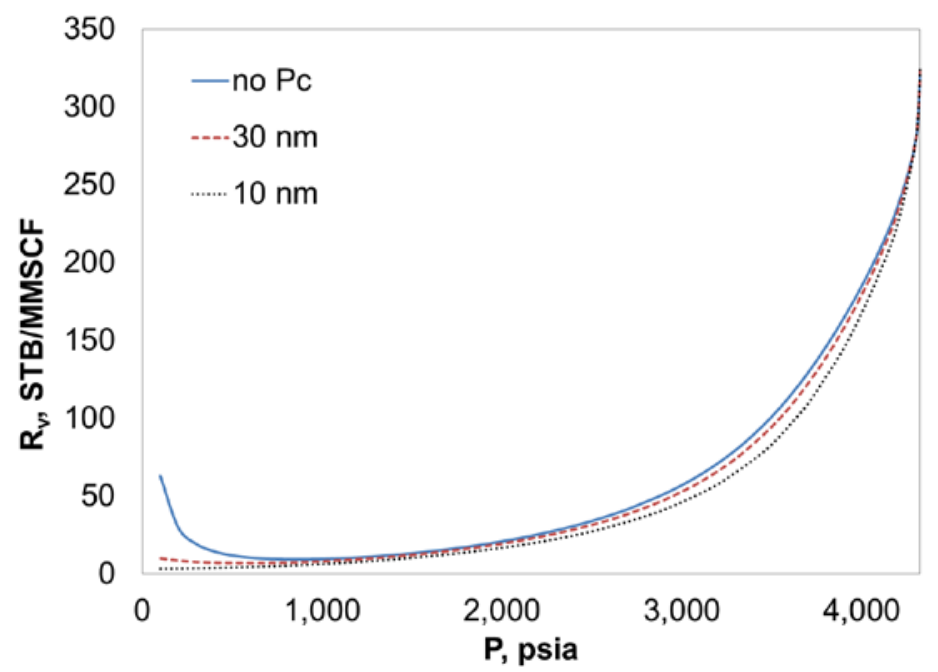

Figure 7- Volatile oil-gas ratio change with oil pressure and effective pore radius up to the critical point at 4325 psia. 
In Figure 8 we show the gas-oil interfacial tension of Bakken fluid as a function of pressure for the case of very large pore sizes and hence negligible capillary pressure. We calculated interfacial tensions to the extrapolated critical point at 4,325 psia based on the Macleod and Sugden correlation, Eq.(8).

$\sigma=\left[\sum_{i} \bar{\chi}_{i}\left(x_{i} \bar{\rho}^{L}-y_{i} \bar{\rho}^{V}\right)\right]^{4}$

$\bar{\chi}_{i}$ is the parachor of component $i$ and $\bar{\rho}^{L}$ and $\bar{\rho}^{V}$ are molar densities of liquid and gas phase respectively. All eight components of the Bakken fluid characterization are included in the flash calculations to determine the densities for input to the Macleod and Sugden correlation.

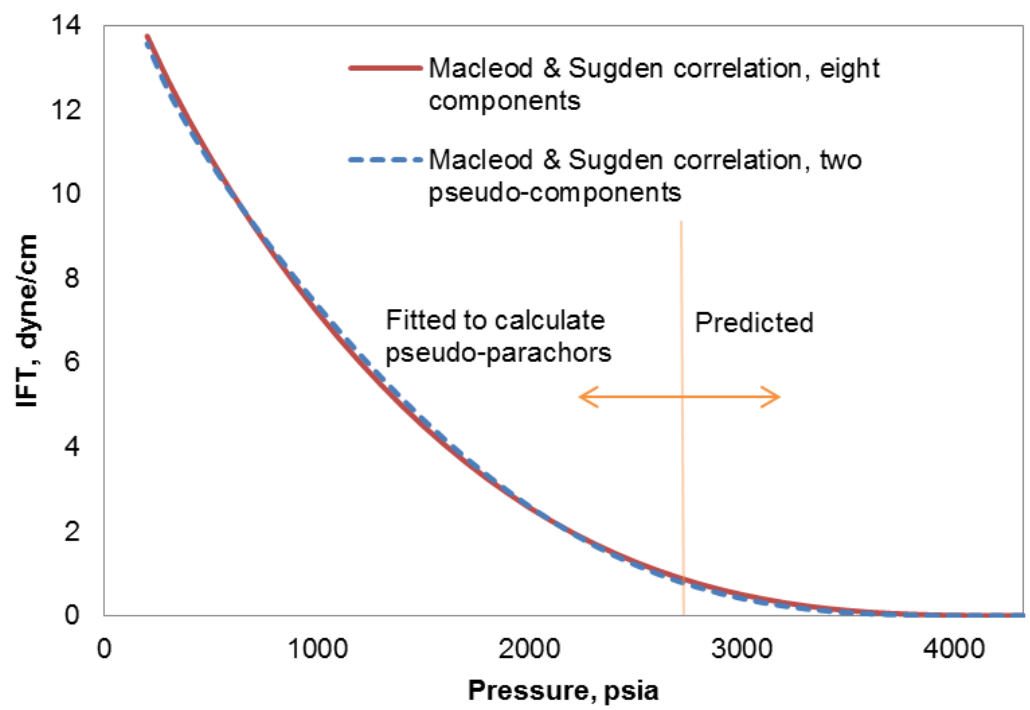

Figure 8- Interfacial tension calculated from the Macleod and Sugden correlation compared to those calculated from the two psuedocomponent black-oil model.

Because capillary pressure can impact the black-oil and volatile-oil properties, a match must be made between the flash calculation IFT (interfacial tension) values and the two-hydrocarbon pseudocomponent model. We adjusted the Macleod and Sugden correlation as follows,

$\sigma=\left[\chi_{o}\left(\omega_{o o} \rho_{o}-\omega_{o g} \rho_{g}\right)+\chi_{g}\left(\omega_{g o} \rho_{o}-\omega_{g g} \rho_{g}\right)\right]^{4}$

where the densities are not molar densities but mass densities. The mass fractions and phase densities are calculated as functions of pressure, but the oil and gas component pseudo-parachors are unknown in Eq. (9). Thus, we performed a linear regression to tune the parachor values to achieve the best match with the multi-component fluid (tuned values were 2.80 for the oil pseudo-parachor and 6.12 for gas pseudo-parachor). The match is excellent as is shown in Figure 8 and both of them predict zero interfacial tension at the critical point as they must.

The liquid and gas phase mass fractions calculated from Eqs. (6) and (7) are used to generate a pressure-composition (PX) diagram of the binary pseudocomponent black-oil phase behavior (see Figure 9). Unlike multicomponent fluids with three or more components, the critical point is at the top of this diagram owing to the differential approach described previously to determine the tie lines. In that approach, the liquid composition becomes lighter, while the equilibrium gas composition becomes heavier until they merge at the top of the two-phase region. 


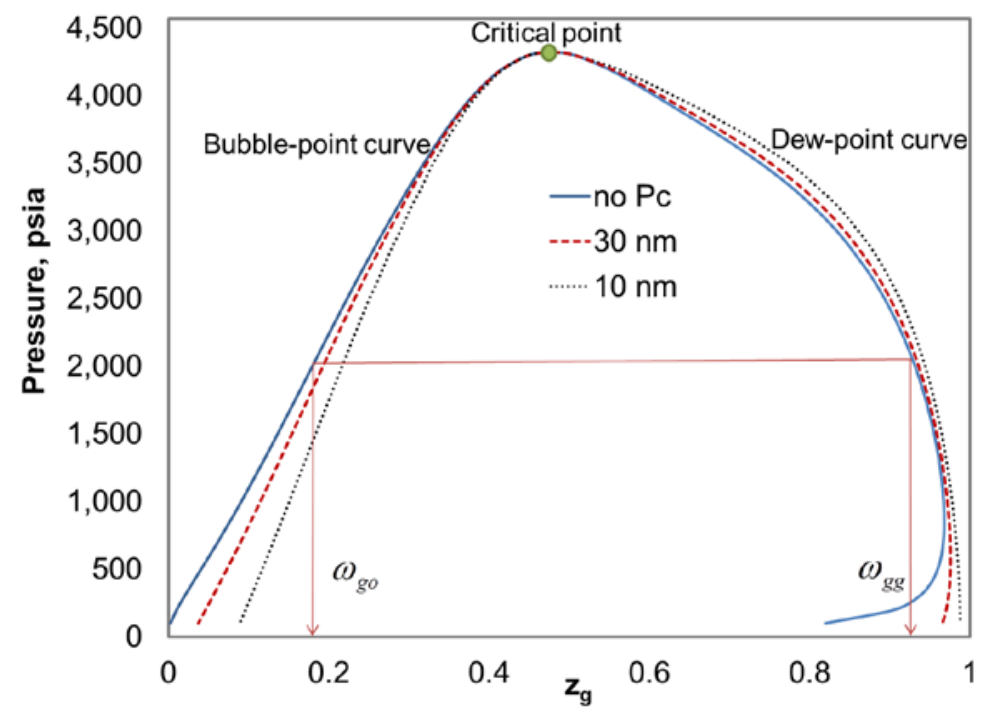

Figure 9- Pressure-composition plot of gas component for three different effective pore sizes.

Figure 9 shows that the bubble-point is suppressed by capillary pressure, while the dew-point is increased in line with previous research (Nojabaei et al. 2013). A "dead" oil composition exists at standard pressure and temperature, which is taken to be the oil pseudocomponent in this diagram (even though this diagram is at constant reservoir temperature). Table 1 gives the eight-component compositions of the original bubble-point oil and gas, surface gas, dead oil, and critical fluid for the Bakken fluid.

Table 1- Composition of various gases and oils

\begin{tabular}{|l|c|c|c|c|r|r|r|r|}
\hline & $\mathrm{C}_{1}$ & $\mathrm{C}_{2}$ & $\mathrm{C}_{3}$ & $\mathrm{C}_{4}$ & $\mathrm{C}_{5}-\mathrm{C}_{6}$ & $\mathrm{C}_{7}-\mathrm{C}_{12}$ & $\mathrm{C}_{13}-\mathrm{C}_{21}$ & $\mathrm{C}_{22}-\mathrm{C}_{80}$ \\
\hline Original bubble-point oil & 0.3674 & 0.1489 & 0.0933 & 0.0575 & 0.0641 & 0.1585 & 0.0733 & 0.0370 \\
\hline Original bubble-point gas & 0.6521 & 0.1676 & 0.0783 & 0.0371 & 0.0285 & 0.0336 & 0.0028 & $1.5 \mathrm{E}-05$ \\
\hline Surface gas & 0.5454 & 0.2177 & 0.1298 & 0.0686 & 0.0356 & 0.0029 & $1.6 \mathrm{E}-07$ & $6.8 \mathrm{E}-17$ \\
\hline Dead oil & 0.0028 & 0.0078 & 0.0188 & 0.0348 & 0.1224 & 0.4771 & 0.2234 & 0.1129 \\
\hline Critical point fluid at 4,325 psia & 0.5234 & 0.1598 & 0.0868 & 0.0479 & 0.0468 & 0.0939 & 0.0316 & 0.0097 \\
\hline
\end{tabular}

We compare the pressure-composition diagram based on our method of extrapolation to those from two other previously mentioned methods of extrapolation for the case of zero capillary pressure. As described in the introduction, one approach is the linear extrapolation of $K$-values on a log-log plot to obtain $K$-values equal to 1.0 at the critical point, which we referred to as the method of Singh et al. (2007). The other approach adds surface gas to the mixture to achieve a larger bubble-point pressure. We applied both of these methods to extrapolate the solution gas-oil and volatile oil-gas ratios so that the mass fractions can be calculated. The results of these three methods, including our differential approach, are plotted on the PX diagram shown in Figure 10. As shown, our approach is the only method that gives a visibly smooth curve all the way to the critical point. Singh et al.'s method gives two linear curves, which meet at the critical point. To apply Singh et al.'s method, we assumed that the convergence pressure is the same as the calculated extrapolated critical pressure. In their method, the choice of critical pressure does not affect the linear shape of the curve; it can only move the critical point up or down. For the approach where surface gas is added, the procedure was stopped when the gas and liquid phase compositions for the gas pseudocomponent became the same. This point does not reflect the correct critical point since interfacial tension is not zero there. 


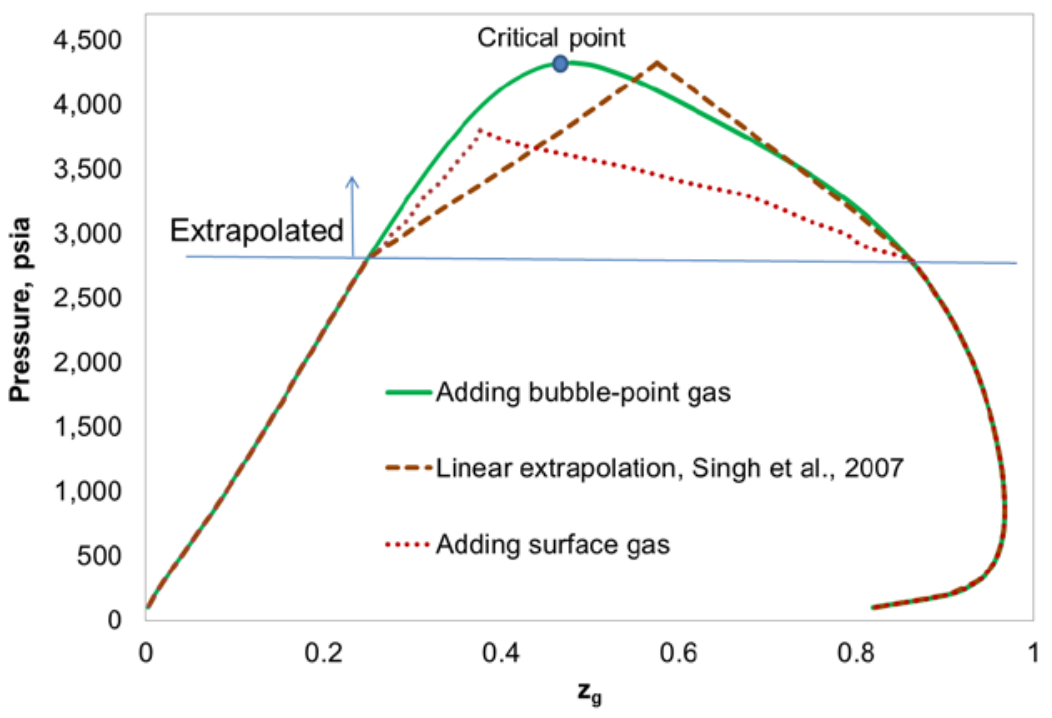

Figure 10- Pressure-composition plot of gas component for the no capillary pressure case using three different extrapolation approaches. The differential approach used in this paper is visibly the best.

Next, we varied the value of $\alpha$ in Eq. (3) to see how this parameter impacts the extrapolated phase behavior. As $\alpha$ decreases, the PX diagram becomes smoother and the results more consistent since the process is based on infinitesimal additions (or subtractions) of equilibrium gas. Figure 11 shows that using different $\alpha$ values results in a change in the extrapolated critical pressure. A value of $\alpha$ extrapolated to zero should give a critical pressure of $4,297 \mathrm{psia}$, while the critical pressure is 4,325 psia using $\alpha=0.1$. These two values are only 28 psi apart, but these small differences could be used to adjust slightly the critical point pressure by changing the value of $\alpha$ if needed, say to match the minimum miscibility pressure (MMP) for a gas flood.

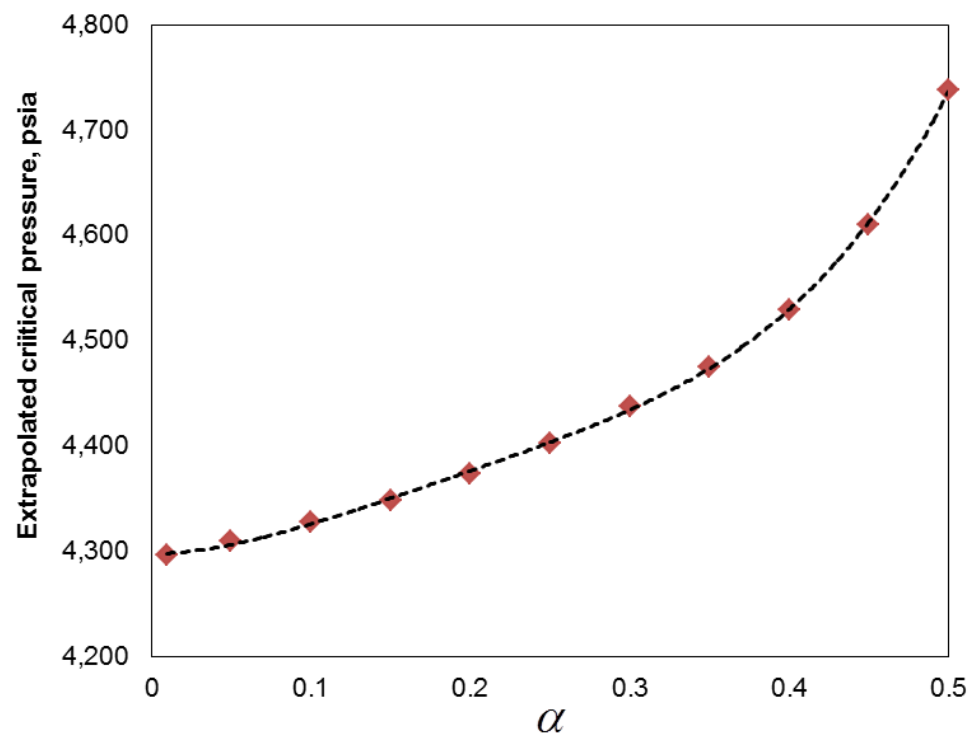

Figure 11- Extrapolated critical pressures with different values of $\alpha$ in Eq. (3).

Last, we considered how to modify the compositionally extended black-oil phase behavior to model gas injection, where gases such as methane, produced gas, or $\mathrm{CO}_{2}$ may be used. One possibility is to reinject the gas pseudocomponent so that there are only two hydrocarbon pseudocomponents. This would correspond to recycling of the produced (surface gas) without removing the intermediate components in the gas (such as ethane, propane, butane). The use of only two hydrocarbon components would be acceptable if the actual MMP from slim-tube experiments or other fluid characterization methods for displacement of bubble-point oil (original oil composition) by surface gas is close (or better yet equal) to the critical pressure of the pseudo-binary PX diagram. 
We calculated MMPs for displacements based on the original eight-component Bakken fluid using our in-house flash calculation package PennPVT (2013), where MMPs are determined with the multiple mixing-cell approach developed by Ahmadi and Johns (2011). The extrapolated critical pressure (about 4,300 psia) from the PX diagram is substantially greater than the MMP of 2,706 psia from PennPVT, indicating that the surface gas is much richer in intermediate components than the equilibrium gases in Figure 10 that were used to generate the two-phase envelope and critical point. The compositions of these fluids are shown in Table 1. Because of the large difference in MMP, it would not be accurate to use the surface gas to represent a miscible or near miscible displacement by the phase behavior given in the PX diagram of Figure 10. Thus, more than two hydrocarbon components would be required for this injection gas.

Table 2 gives MMPs for other gas-oil displacements as calculated by PennPVT. For example, the MMP is about 3,907 psia for injection of methane to displace original bubble-point oil. This value is close to the critical point pressure of the pseudo-binary PX diagram in Figure 10 since the equilibrium gas used to generate that figure was relatively light compared to the surface gas. Thus, if pure methane were injected one could use the phase behavior in Figure 10 to model the miscible process, although the MMP value would be in error by about $10 \%$.

Table 2- MMPs for different oils and gases caculated using multiple mixing cell method (Ahmadi and Johns, 2011)

\begin{tabular}{|l|l|}
\hline Surface gas and original bubble-point oil & $2706 \pm 36$ psia \\
\hline Methane and original bubble-point oil & $3907 \pm 2$ psia \\
\hline Methane and dead oil & $5768 \pm 7$ psia \\
\hline $\mathrm{CO}_{2}$ and original bubble-point oil & $2230 \pm 50$ psia \\
\hline $\mathrm{CO}_{2}$ and dead oil & $3061 \pm 19$ psia \\
\hline
\end{tabular}

Table 2 also gives the MMP values for injection of $\mathrm{CO}_{2}$. The MMP for displacement of original bubble-point oil by $\mathrm{CO}_{2}$ is about 2,230 psia, while the "hypothetical" displacement of dead oil by $\mathrm{CO}_{2}$ gives an MMP around 3,061 psia. The large MMP for the displacement of dead oil is consistent again with the dead oil containing more heavy components than the original bubble-point oil. Although the MMP for displacement of dead oil is not practical, this value should be approximately the same as the critical pressure of the corresponding PX diagram for $\mathrm{CO}_{2}$-dead oil $(3,160$ psia from the PX plot compared to a MMP of 3,061 psia in this case).

The above results indicate that it would be advantageous to introduce a third hydrocarbon (or nonhydrocarbon component) to the black- or volatile-oil fluid characterization to model miscible or near miscible gas injection. For example, to model a displacement of original bubble-point oil by methane, one could introduce methane as another gas component keeping the surface gas and dead oil as the two other hydrocarbon pseudocomponents. Displacements by $\mathrm{CO}_{2}$ would require $\mathrm{CO}_{2}$ be a separate third component to match the MMP.

The introduction of a second gas component (or solvent) complicates the black oil model. Compositionally-extended black-oil systems, however, can be easily represented by ternary diagrams as is shown next. Adding a fourth component then amounts to modeling the phase behavior using quaternary diagrams.

Consider first the standard black oil model represented on a ternary diagram. Here, we have three components (water, dead oil, and surface gas) and three phases (aqueous, oleic, and vapor). The aqueous phase consists of pure water component, and as a result, the composition of the aqueous phase is always at the apex of the ternary diagram (the inclusion of gas in water could be easily added, but is not shown here for simplicity). The oil and gas components are not miscible and thus a tie line connects their equilibrium phases (bubble-point oil and dew-point gas) on the oil-gas axis of the ternary diagram. This tie line is also a boundary of the three-phase region. The PX diagram in Figure 10 gives the length of the tie line on the gas-oil axis at various pressures. The three-phase region extends from the water apex to the two equilibrium compositions. Figures 12 shows the change in the ternary phase behavior for the compositionally-extended black-oil model for four different pressures including one close to the critical pressure between the gas and oil pseudo-components. The dashed lines on these figures are tie lines. The figure shows that the three-phase region shrinks with an increase in pressure as is expected. For pressures greater than the critical point, there is just one two-phase region, where only the aqueous and supercritical phases remain. 

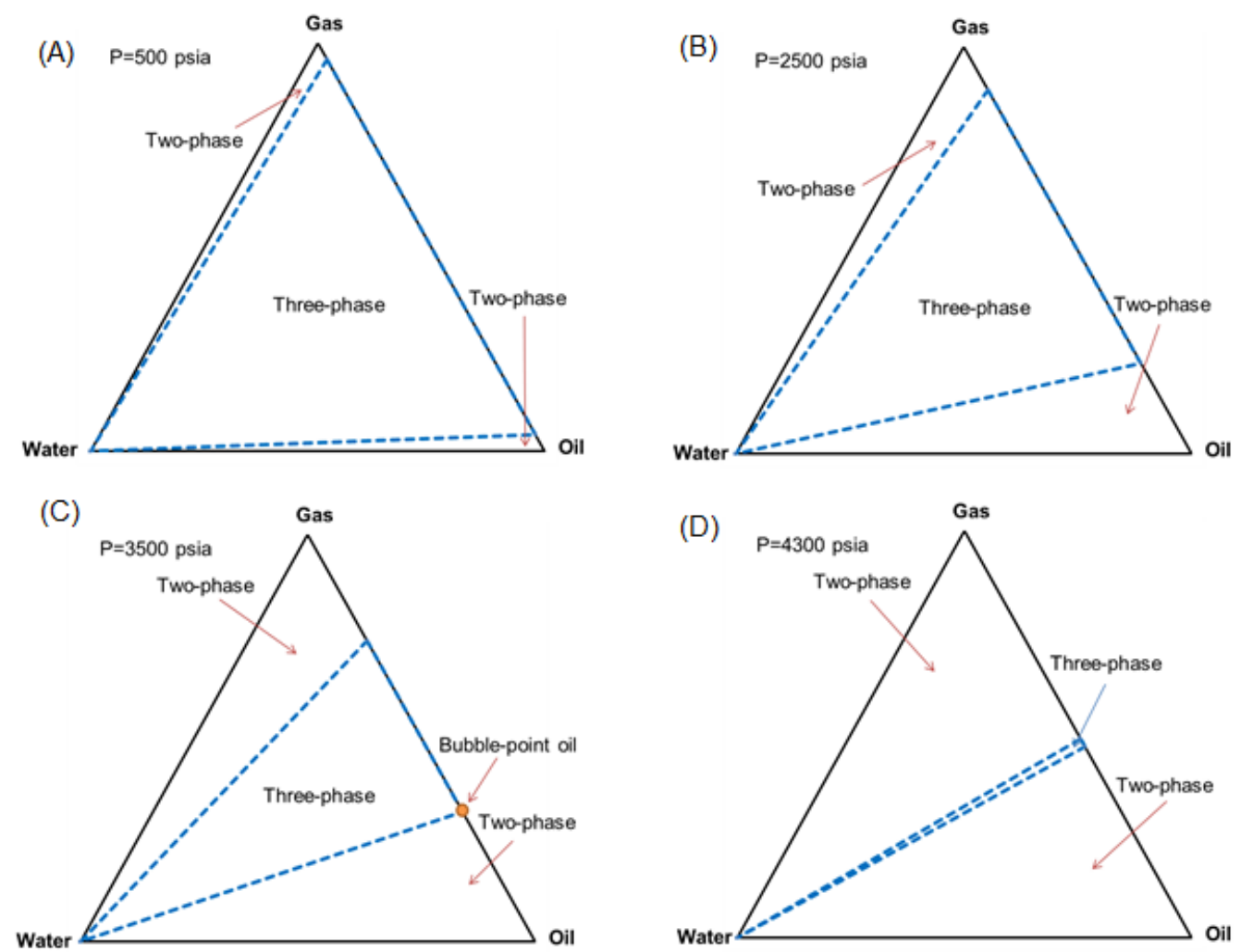

Figure 12- Ternary diagram of three component black-oil system at (A) $P=500$ psia, (B) $P=2500$ psia, $(C) P=3500$ and $(D) P=4300$ psia (just below the critical pressure).

Consider now injection of pure methane to displace the original bubble-point oil at constant reservoir temperature. The oil pseudocomponent is the dead oil composition. We lump the dead oil composition to one single fluid component and then perform negative flash calculations for the dilution of this component with methane at different pressures (see Figures 13). The critical pressure is at the top of these diagrams at a pressure of about $6,470 \mathrm{psia}$. Ideally, this value should be the same as the minimum miscibility pressure (MMP) of methane and eight-component dead oil using PennPVT (or slim-tube measurements). As shown in Table 2 they are within $10 \%$ of each other (6,470 psia compared to 5,768 psia), but they are not exact. Although not done here, one could adjust the properties of the dead oil pseudocomponent by modifying the lumping approach in the PX diagram to achieve a match to the MMP of 5,768 psia.
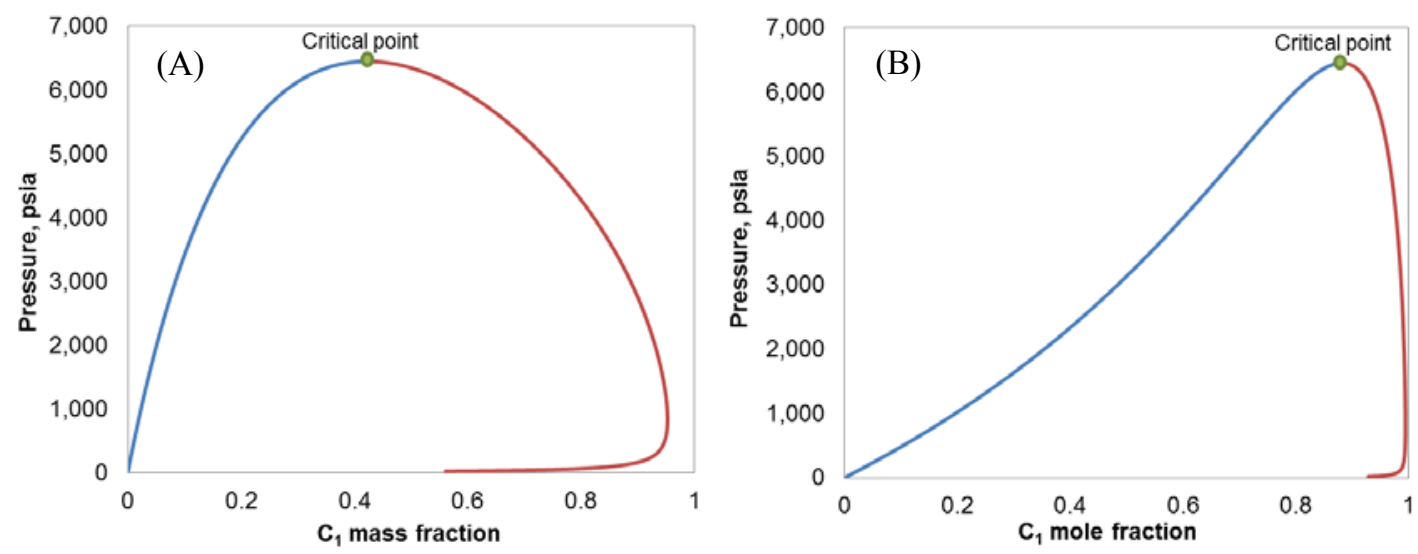

Figure 13- (A) Pressure-mass composition plot, (B) Pressure-composition plot of methane diluted with dead oil

The PX diagram for methane and the dead oil component is a limiting case of the oil-gas- $\mathrm{CH}_{4}$ ternary diagram (corresponding to its oil- $\mathrm{CH}_{4}$ axis), which is one of the sides of the quaternary diagram (for the four-component water-oilgas- $\mathrm{CH}_{4}$ system). The four sides of the quaternary diagram are shown in Figures $\mathbf{1 4}$ and $\mathbf{1 5}$ at a pressure of 2,500 psia, which is below the critical pressure of the oil-gas axis, and for a pressure of 4,325 psia, which is equal to its critical pressure. 
The critical point locus in the oil-gas- $\mathrm{CH}_{4}$ ternary diagram is approximately linear with pressure and composition. For consistency, we also used lines to represent the bubble-point and dew-point curves in that face of the quaternary diagram. The $K$-values inside the ternary diagrams are estimated by assuming that the tie line extends to the dead oil apex and through the overall composition specified (Tang and Zick, 1993). This approach also allows for mixtures of $\mathrm{CH}_{4}$ and produced gas to be injected. The critical locus is shown in the figures by a dotted red line while the blue dashed lines are tie lines. These flattened quaternary diagrams are based on mass fractions and not molar compositions, although molar values can also be used.

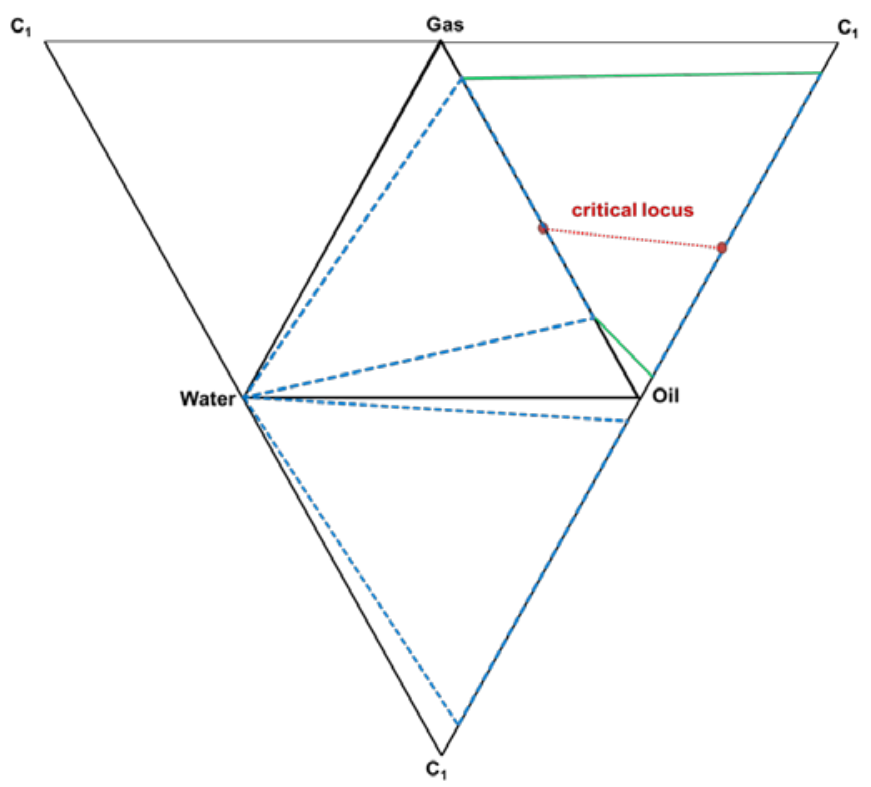

Figure 14- Flattened quaternary diagram of four-component extended black-oil system with methane at $P=2500$ psia.

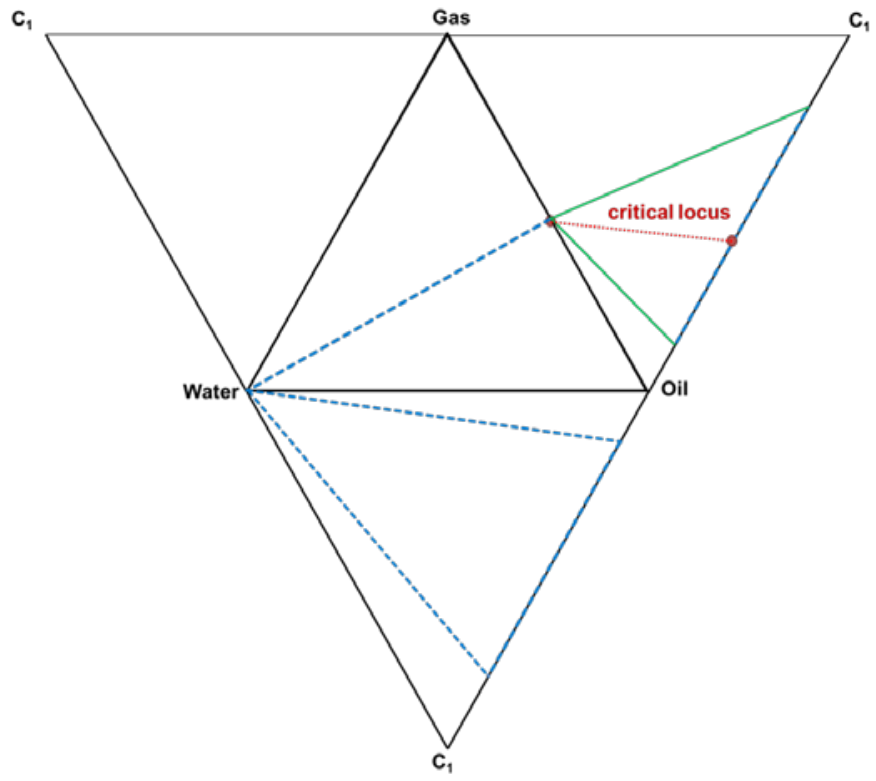

Figure 15- Flattened quaternary diagram of four-component extended black-oil system with methane at $\mathrm{P}=4325$ psia.

Next, we substitute methane by $\mathrm{CO}_{2}$ and repeat the process. Displacement of dead oil by $\mathrm{CO}_{2}$ will result in a significantly lower critical pressure and MMP as shown in Figures 16, and also in Table 2. These two values agree well (eight component MMP of 3,061 psia compared to 3,160 psia on the PX diagram). Again, one could tune the properties of the dead oil pseudocomponent in the PX diagram to match the actual MMP. Figures 17 and 18 give the sides of the quaternary diagram for the four component oil-gas-water- $\mathrm{CO}_{2}$ system at two different pressures of 2,500 psia and 3,160 psia (dead oil- $\mathrm{CO}_{2}$ critical pressure). 

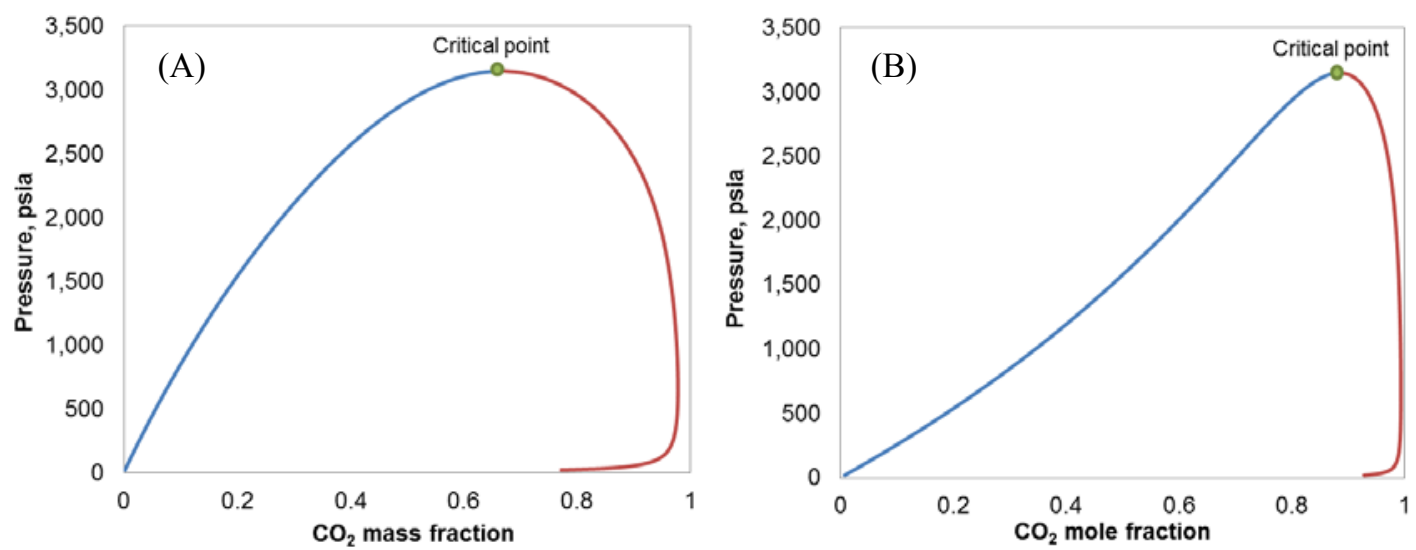

Figure 16- (A) Pressure-mass composition plot, (B) Pressure-composition plot of $\mathrm{CO}_{2}$ diluted with dead oil

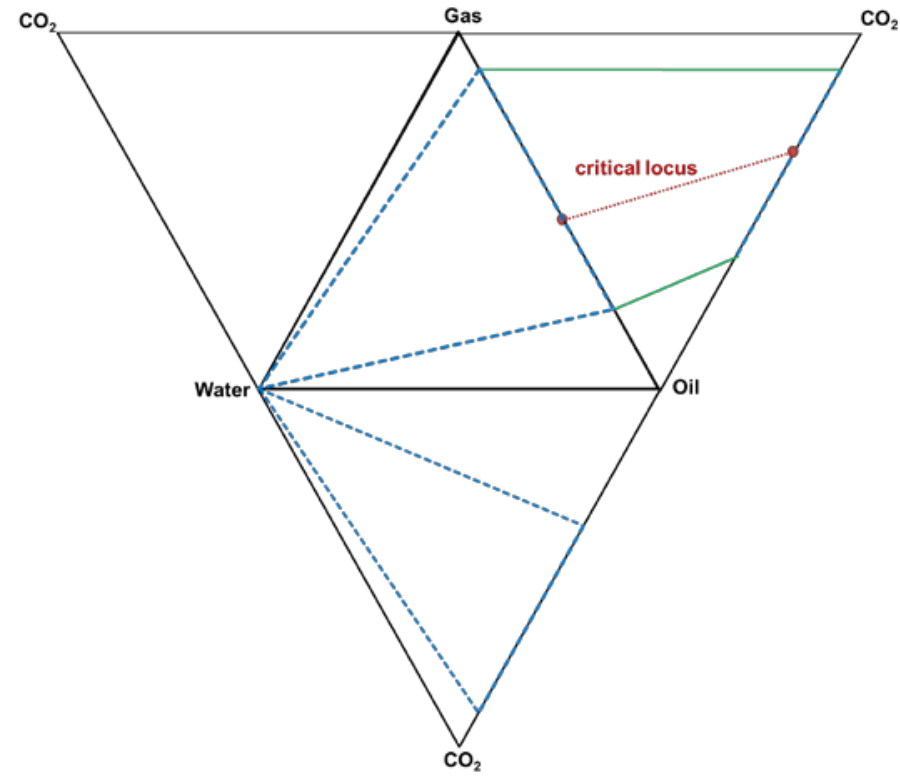

Figure 17- Flattened quaternary diagram of four-component extended black-oil system with $\mathrm{CO}_{2}$ at $\mathrm{P}=2500$ psia.

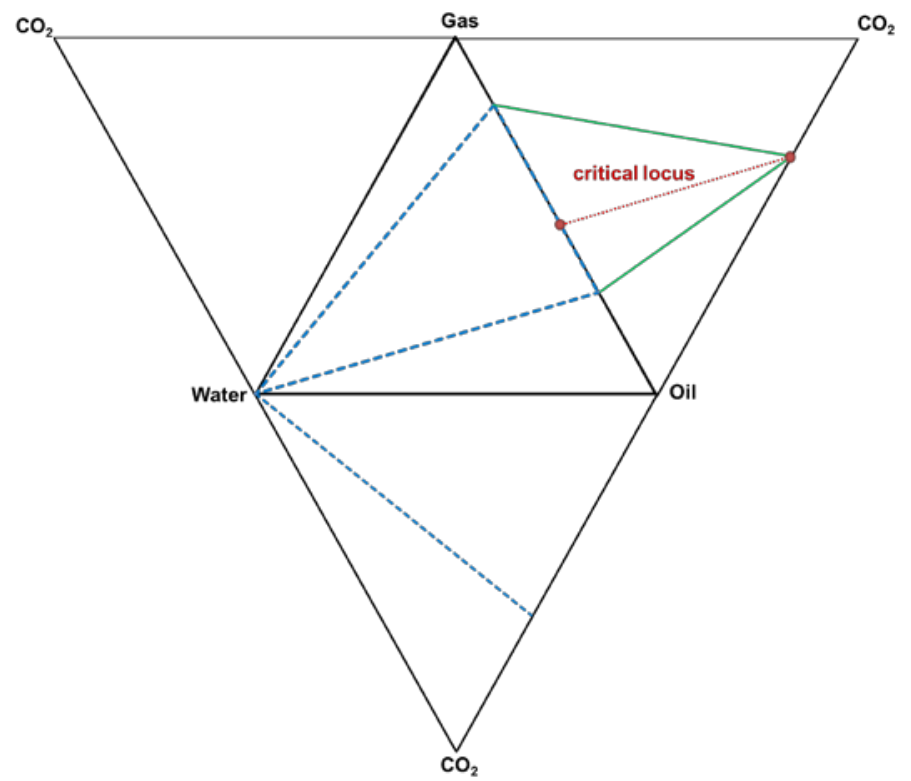

Figure 18- Flattened quaternary diagram of four-component extended black-oil system with $\mathrm{CO}_{2}$ at $\mathrm{P}=3160 \mathrm{psia}$. 
The displacements by methane or $\mathrm{CO}_{2}$ would require adding a fourth mass conservation equation in the formulation. This would not greatly increase the computational cost because the $K$-values are known. Only Rachford-Rice iteration (Rachford and Rice, 1952) would be required. It may also be possible to include a fifth component in this model (say methane and $\mathrm{CO}_{2}$ ), but the pseudocomponent for the dead oil would have to be tuned to give the correct MMP in both the oil$\mathrm{CO}_{2}$ and oil- $\mathrm{CH}_{4} \mathrm{PX}$ diagrams.

\section{Slim-tube Simulation for Bakken Oil with Extended Black-oil Approach}

In the previous section it was shown that the extrapolated critical point is at the top of the phase envelope for a pseudo-binary mixture. In this section, we show how we can use these extrapolated black oil fluid properties to perform a slim-tube simulation for Bakken oil when the injection gas is produced gas. The simulation model is presented in Figure 19. Gas is injected through the first grid block on the left.

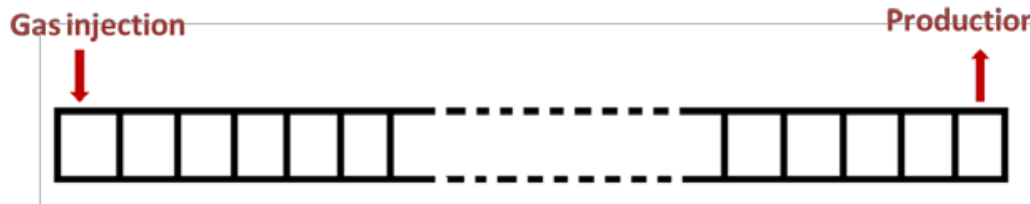

Figure 19- Schematic of the slim tube simulation example

We use an explicit Euler approach for performing slim-tube simulations. Pressure is fixed and compositions are solved explicitly. Fractional flow curves are used to calculate the change in compositions. Initial oil is the original Bakken oil composition but based on two pseudo-components. The simplified mass conservation equation for component $i$ is solved to calculate compositions where no water is used, and there is no volume change on mixing.

$\frac{\partial C_{i}}{\partial t_{D}}+\frac{\partial F_{i}}{\partial x_{D}}=0$.

In Eq.(10), $C_{i}$ is the volumetric composition of component $i$ and $F_{i}$ is the flux term, which are obtained as follows:

$C_{i}=\sum_{j=1}^{N_{p}} C_{i j} S_{j}$

and

$F_{i}=\sum_{j=1}^{N_{p}} C_{i j} f_{j}$.

Fractional flow is given by the standard definition as,

$f_{j}=\frac{\lambda_{j}}{\sum_{j=1}^{N_{p}} \lambda_{j}}=\frac{k_{r j} / \mu_{j}}{\sum_{j=1}^{N_{p}} k_{r j} / \mu_{j}}$.

Slim-tube simulations are performed for two oil and gas pseudo-components. For simplicity, oil and gas relative permeabilities are calculated as:

$k_{r o}=\left(1-S_{g}-S_{o r}\right)^{2}, k_{r g}=S_{g}^{2}$

All fluid properties (densities and viscosities), saturations and $K$-values are calculated explicitly using black-oil fluid properties. No flash calculation is done in the slim-tube simulations. 100 grid blocks are used and $1.2 \mathrm{PV}$ gas is injected.

Gas saturation and gas composition profiles from the simulations for nine different pressures are shown in Figures 20 and 21 respectively. There are two fronts as is expected for a binary displacement, a leading front, followed by a trailing evaporation front. As pressure increases, the saturation fronts merge. That is, for pressures greater than pseudo-critical pressure (4325 psia for Bakken) the displacement becomes first contact miscible and flow is piston-like (the fronts merge when the leading shock slows down and the trailing front speeds up). Pseudo critical pressure is the MMP as there are only 
two components. Although not shown, composition fronts become sharper as number of grid blocks is increased.

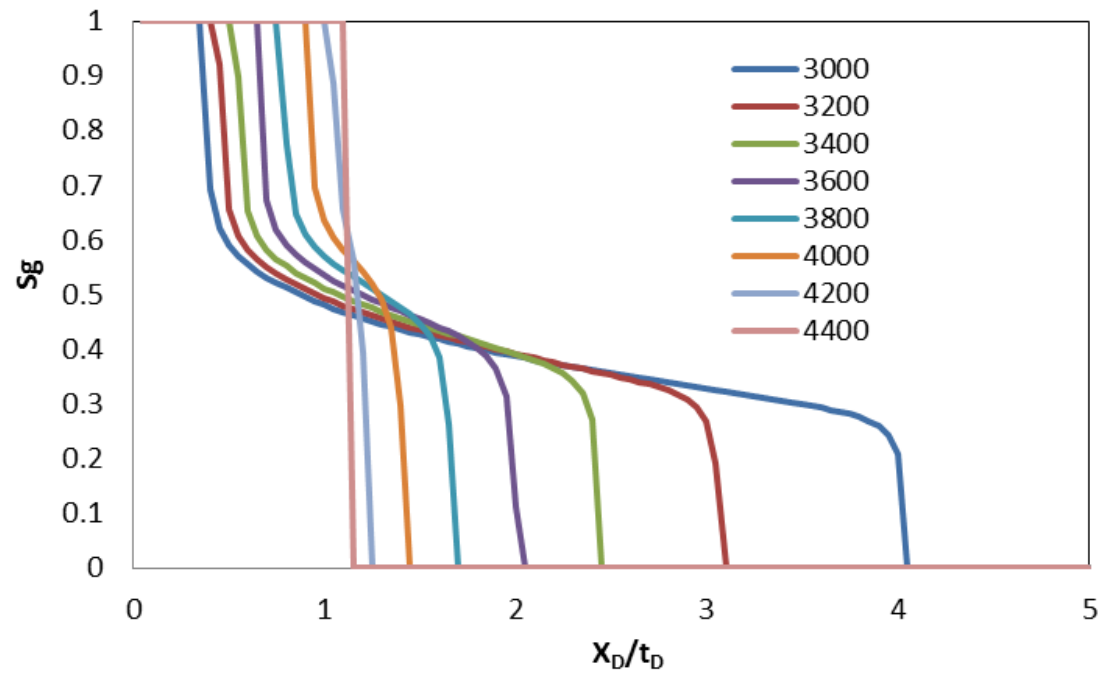

Figure 20-Saturation profile as a function of dimensionless velocity for different pressures

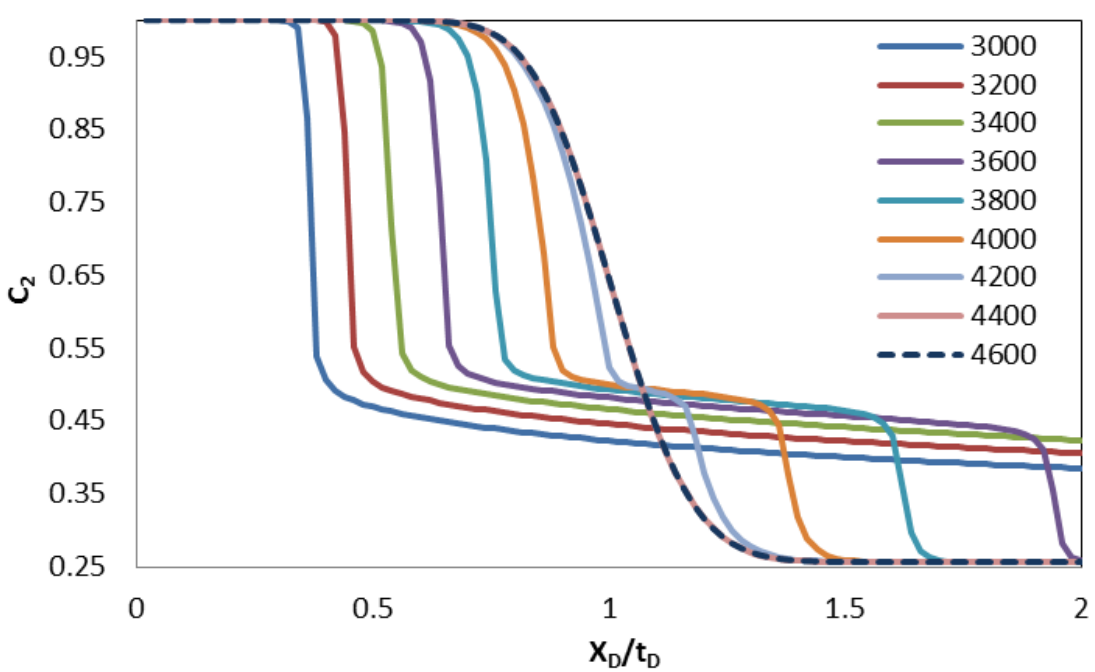

Figure 21- Gas overall composition as a function of dimensionless velocity for different pressures

Oil component recovery is plotted at 1.0 pore volumes injected (PVI) in Figure 22. MMP can be determined from the bend of the recovery curve, and it is about 4300 psia in this example, which agrees well with the MMP of the original oil and reservoir gas. Calculated recovery depends on the grid resolution. For 100 grid blocks, numerical dispersion prevents an exact answer for MMP from the recovery data. Although not done here, extrapolation of the recovery curve to an infinite number of grid blocks could be used to account better for numerical dispersion (Stalkup, 1987). 


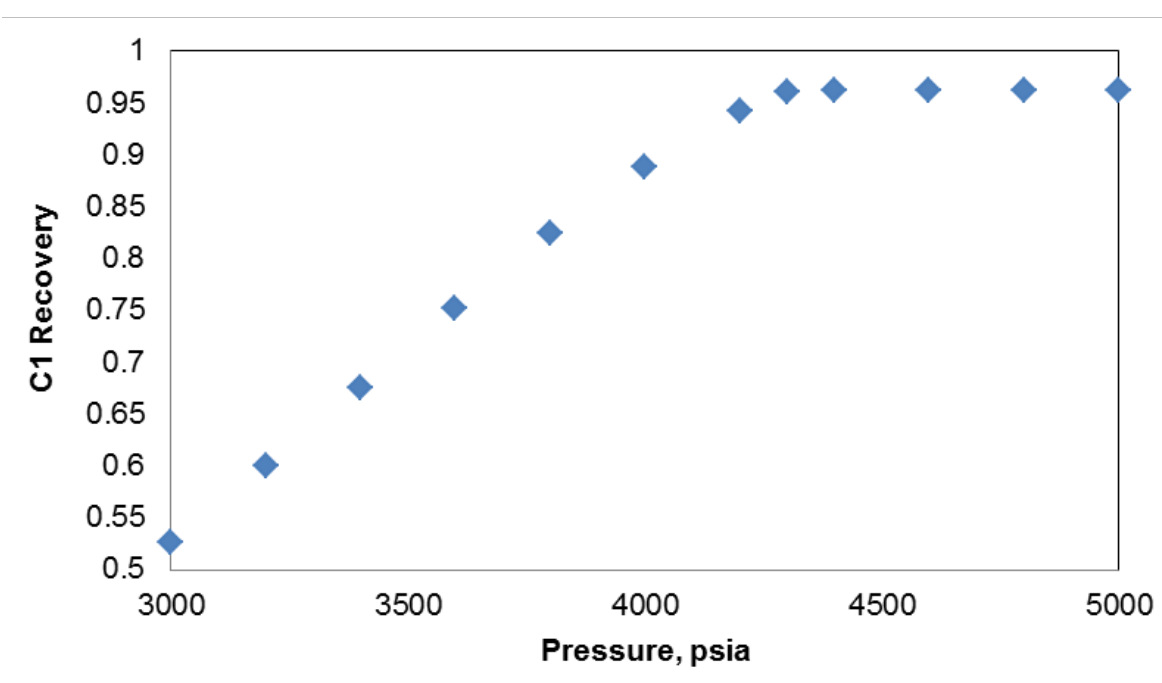

Figure 22- Oil component recovery as a function of pressure at 1.0 PVI

\section{Summary and Conclusions}

We demonstrated a consistent approach to calculate variable black- and volatile-oil fluid properties below and above the original bubble-point pressure where the saturation pressures changes with both oil composition (solution gas-oil ratio) and capillary pressure (effective pore size). The approach shown determines the fluid properties for a variable bubble-point by adding fractions of the equilibrium gas composition with its bubble-point oil to achieve a new larger bubble-point pressure. This differential procedure continues until a critical point is reached.

The key conclusions of this research are:

- The approach gives continuous extrapolation of all black- and volatile-oil properties by adding small increments of equilibrium bubble-point gas to the critical point. As these increments decrease, the results are more reliable.

- An increase in capillary pressure decreases the bubble-point pressure at constant oil composition, while an increase in the solution gas-oil ratio increases the bubble-point pressure at constant capillary pressure. These effects can be included simultaneously in a compositionally-extended black- or volatile-oil formulation.

- The two pseudo-component correlation developed here predicts IFTs very close to the values calculated from Macleod and Sugden correlation for the eight-component Bakken fluid. Both of these correlations give zero IFT at the extrapolated critical point.

- The new model could be used for both multicontact miscible and immiscible gas injection by matching the MMPs for the key binary endpoints. No flash calculations with a cubic equation-of-state are needed during simulation.

The approach could be extended to gas condensate reservoirs, and could potentially allow for a fourth hydrocarbon component as long as the binary endpoint MMPs are matched.

\section{Acknowledgements}

This research was partly sponsored by the Unconventional Natural Resources Consortium (UNRC) and the Enhanced Oil Recovery Research Consortium at the EMS Energy Institute at the Pennsylvania State University. Dr. Russell T. Johns is the Chair of the Petroleum and Natural Gas Engineering program in the John and Willie Leone Family Department of Energy and Mineral Engineering at Penn State. He also holds the CMG Foundation Chair in fluid behavior and rock interactions at Penn State, and the Beghini faculty fellowship in Petroleum and Natural Gas Engineering.

\section{Nomenclature}

$\begin{array}{lll}f & = & \text { fugacity, } \text { sia } \\ K & = & \text { equilibrium ratio } \\ N_{p} & =\text { number of phases, excluding water } \\ P & = & \text { pressure, } \text { psia } \\ P_{c} & = & \text { capillary pressure, } p s i \\ r & = & \text { pore radius, } n m \\ R_{S o} & = & \text { solution gas-oil ratio, } S C F / S T B \\ R_{v} & = & \text { volatile oil-gas ratio, } S T B / M M S C F\end{array}$




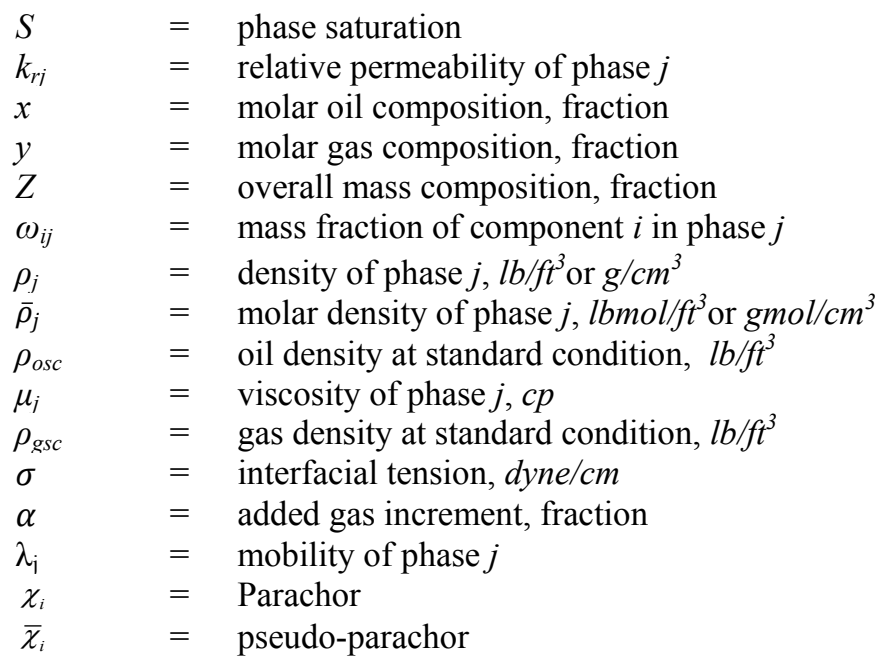

\section{Unit conversion}

Field Units SI Units

Density

Gas production

Interfacial tension

Length

Oil production

Permeability

Pressure

Solution gas-oil ratio

Viscosity

Volatile oil ratio $l b / f t^{3}$

SCF

dyne/cm

$f t$

STB

md

Psia

SCF/bbl

$=0.001$ Pascal.sec

$\mathrm{bbl} / \mathrm{MMSCF} \quad=0.159 /(0.028 \mathrm{e} 6) \mathrm{m}^{3} / \mathrm{m}^{3}$

$\begin{array}{ll}= & 16.018 \mathrm{~kg} / \mathrm{m}^{3} \\ = & 0.028 \mathrm{~m}^{3} \\ = & 0.001 \mathrm{~N} / \mathrm{m} \\ = & 0.305 \mathrm{~m} \\ = & 0.159 \mathrm{~m}^{3} \\ = & 9.869 \mathrm{e}-16 \mathrm{~m}^{2} \\ = & 6894.757 \text { Pascal } \\ = & 0.028 / 0.159 \mathrm{~m}^{3} / \mathrm{m}^{3} \\ = & 0.001 \text { Pascal.sec } \\ = & 0.159 /(0.028 \mathrm{e} 6) \mathrm{m}^{3} / \mathrm{m}^{3}\end{array}$

\section{References}

Ahmadi K., Johns, R.T., Multiple-mixing-cell method for MMP calculations, SPE Journal, volume16, issue 04, 2011.

Ayirala S.C., Rao D.N., A new parachor model to predict dynamic interfacial tension and miscibility in multicomponent hydrocarbon systems, Colloid and Interface Science, 299, 321-331, 2006.

Dong C., Hoffman B.T., Modeling gas injection into shale oil reservoirs in the Sanish field, North Dakota, presented at Unconventional Resources Technology Conference, 12-14 August, Denver, Colorado, 2013.

Dandekar A.Y., Petroleum reservoir rock and fluid properties, second edition, CRC Press, Taylor and Francis group, 2013.

Macleod, D.B., On a relation between surface tension and density, Trans., Faraday Soc., J. Chem. Soc., volume 125, 38-42, 1923.

McCain W.D., Spivey, J.P., Extrapolation of laboratory measured black oil and solution gas fluid properties for variable bubblepoint simulation, presented at SPE Annual Technical Conference and Exhibition, Houston, Texas, 1999.

Montel F., Quettier L., Getting the best from the black-oil approach for complex reservoir fluids, presented at SPE Annual Technical Conference and Exhibition, 26-29 September, Houston, Texas, 2004.

Nojabaei B., Johns T.R., Chu L., Effect of capillary pressure on phase behavior in tight rocks and shales, SPEREE, volume16, 281-289, 2013.

Nojabaei B., Siripatrachai N., Johns R.T., Ertekin T, Effect of saturation dependent capillary pressure on production in tight rocks and shales: a compositionally-extended black oil formulation, presented at the SPE Eastern Regional Metting, Charlston, West Virginia, 2014.

Pedersen K.S., Christensen P.L., Phase behavior of petroleum reservoir fluid, CRC Press, Taylor \& Francis Group, 2007.

Peng, D. Y., and Robinson, D. B., A new two-constant equation of state, industrial and engineering chemistry: fundamentals, volume 15, 
59-64, 1976.

PennPVT toolkit, Gas flooding ioint industry project, Director: Dr. Russell T. Johns, EMS Energy Institute, The Pennsylvania State University, University Park, PA, 2013.

Rachford, H.H. and Rice, J.D.: "Procedure for use of electrical digital computers in calculating flash vaporization hydrocarbon equilibrium," Journal of Petroleum Technology, Sec. 1, p. 19, Oct. 1952.

Shoaib S., Hoffman B.T., CO2 flooding the Elm Coulee field, presented at SPE Rocky Mountain Petroleum Technology Conference, Denver, Colorado, 2009.

Singh K., Fevang O., Whitson C.H., Consistent black-oil PVT table modification, presented at SPE Annual Technical Conference and Exhibition, Anaheim, California, 2007.

Siripatrachai N., Ertekin T., Johns R.T., Compositional simulation of discrete fractures incorporating the effect of capillary pressure on phase behavior, presented at the, SPE Improved Oil Recovery Conference, Tulsa, Oklahoma, 2016.

Stalkup F.I., Displacement behavior of the condensing/vaporizing drive process, presented at SPE Annual Technical Conference and Exhibition, Dallas, Texas, USA, 1987.

Sugden S., The variation of surface tension with temperature and some related function, J. Chem. Soc, Vol. 125, 32-41, 1924.

Tang D.E. , Zick A.A., A new limited compositional reservoir simulator, presented at SPE Symposium on Reservoir Simulation, New Orleans, Louisiana, 1993.

Todd M.R., Longstaff W.J., The development, testing, and application of a numerical simulator for predicting miscible flood performance, Journal of Petroleum Technology, Volume 24, issue 07, 1972.

Whitson, C.H., Sunjerga S., PVT in liquid-rich shale reservoirs, presented at SPE Annual Technical Conference and Exhibition, San Antonio, Texas, 2012.

Wong T.W., Firoozabadi A., Nutakki R., Aziz K., A comparison of two approaches to compositional and black oil simulation, presented at the $9^{\text {th }}$ SPE Symposium on Reservoir Simulation, San Antonio, Texas, 1987. 